NMR and EPR

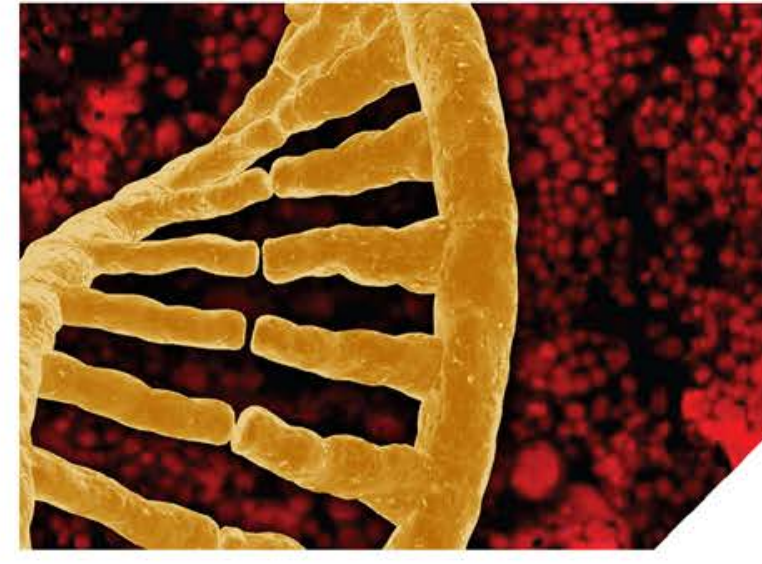

Science Drivers and Technical

Challenges for Advanced

Magnetic Resonance

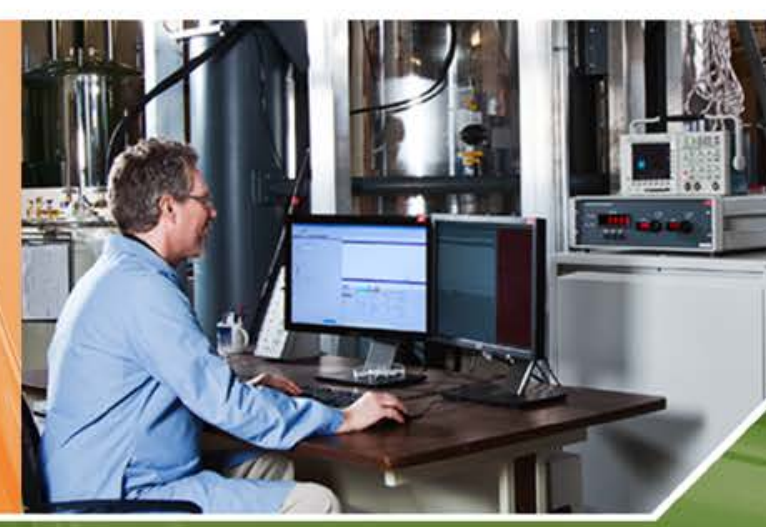

December 7-8, 2011

Report: March 2013

(6)
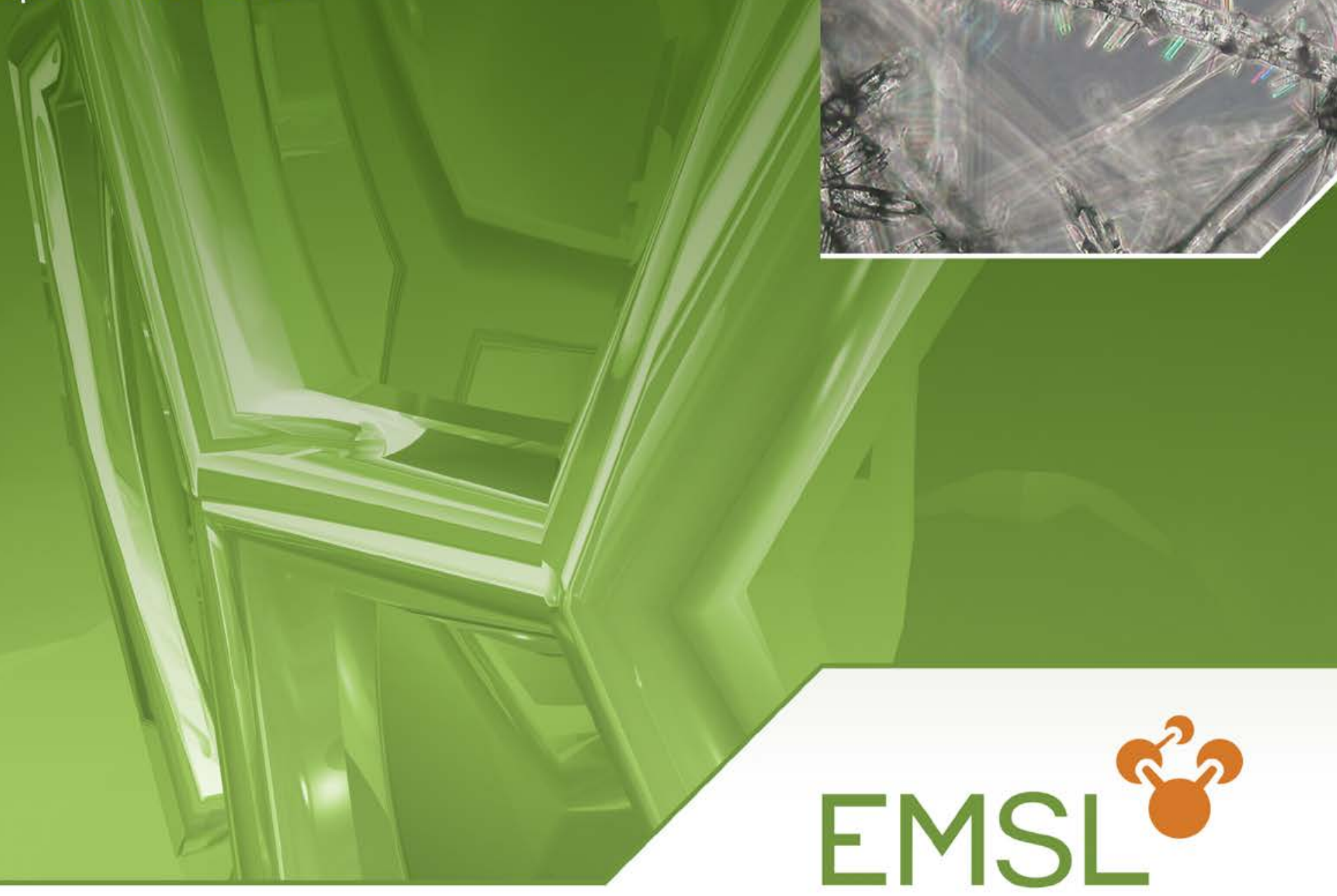


\section{DISCLAIMER}

This report was prepared as an account of work sponsored by an agency of the United States Government. Neither the United States Government nor any agency thereof, nor Battelle Memorial Institute, nor any of their employees, makes any warranty, express or implied, or assumes any legal liability or responsibility for the accuracy, completeness, or usefulness of any information, apparatus, product, or process disclosed, or represents that its use would not infringe privately owned rights. Reference herein to any specific commercial product, process, or service by trade name, trademark, manufacturer, or otherwise does not necessarily constitute or imply its endorsement, recommendation, or favoring by the United States Government or any agency thereof, or Battelle Memorial Institute. The views and opinions of authors expressed herein do not necessarily state or reflect those of the United States Government or any agency thereof.

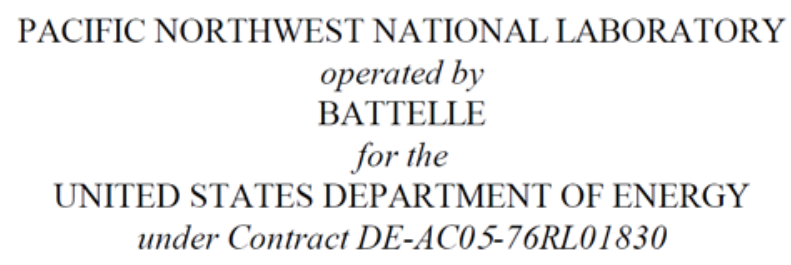

Printed in the United States of America

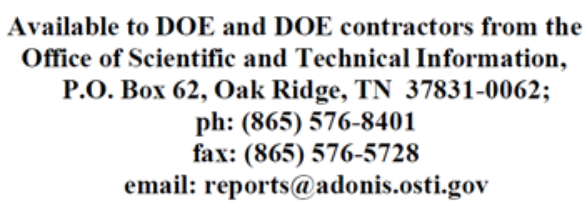

Available to the public from the National Technical Information Service, U.S. Department of Commerce, 5285 Port Royal Rd., Springfield, VA 22161 ph: (800) 553-6847 fax: (703) 605-6900

email: orders@ntis.fedworld.gov online ordering: http://www.ntis.gov/ordering.htm

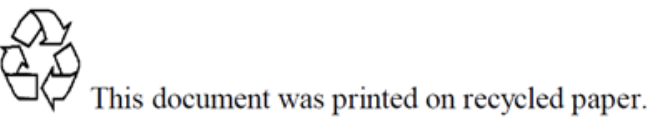




\title{
Science Drivers and Technical Challenges for Advanced Magnetic Resonance
}

\author{
KT Mueller \\ NM Washton \\ M Pruski \\ AS Lipton
}

Report: March 2013

Prepared for the U.S. Department of Energy's

Office of Biological and Environmental Research

under Contract DE-AC05-76RL01830

Pacific Northwest National Laboratory

Richland, Washington 99352 



\section{Executive Summary: Workshop Background, Purpose, and Organization}

Capabilities for nuclear magnetic resonance (NMR) and electron paramagnetic resonance (EPR) spectroscopy are widespread throughout academia, industry, and national laboratories. The Environmental Molecular Sciences Laboratory (EMSL) high-field magnetic resonance user capability at Pacific Northwest National Laboratory (PNNL) is among the largest in the world. At EMSL, scientific researchers from across the United States and around the world are granted instrument time and staff support through a peer-reviewed science proposal process (for more details, visit www.emsl.pnnl.gov/access/calls). At EMSL, users have access to ultra-high-field solution- and solid-state NMR instrumentation, as well as a state-of-the-art high-field EPR system. Through the development and deployment of advanced magnetic resonance techniques, the science and engineering staff at EMSL provide expert scientific advice to users as they pursue fundamental research within U.S. Department of Energy (DOE) mission needs. An engaged community of dozens of additional magnetic resonance scientists, staff, and users at PNNL translates into a venue where a concentrated effort is ongoing in scientific research and problem solving using advanced magnetic resonance technologies.

To discuss and address challenges for the next generation of magnetic resonance experimentation, an exploratory workshop, "Science Drivers and Technical Challenges for Advanced Magnetic Resonance," was held at EMSL on December 7-8, 2011. The workshop was co-organized by Dr. Karl T. Mueller (Laboratory Fellow, EMSL/PNNL) and Dr. Marek Pruski (Senior Scientist, Ames Laboratory). As part of the workshop, participants from throughout the United States and Europe outlined the science drivers and instrumentation demands for high-field dynamic nuclear polarization (DNP) and associated magnetic resonance techniques, discussed barriers to their advancement, and deliberated the path forward for significant and impactful advances in the field.

An introductory lecture and seven scientific talks were presented by leaders in the DNP field and associated sciences, while two in-depth discussions of emerging technologies were led by participants from companies developing cuttingedge instrumentation. High-level discussions took place at four breakout sessions, led by DOE-supported scientists, addressing scientific drivers and potential applications of DNP in biological sciences, biogeochemistry, materials science, and catalysis/energy sciences. A full list of workshop participants and a detailed workshop agenda are included herein as Appendix B and Appendix C, respectively. 



\title{
Acronyms and Abbreviations
}

\author{
DNP \\ dynamic nuclear polarization \\ DOE \\ U.S. Department of Energy \\ EMSL \\ Environmental Molecular Sciences Laboratory \\ EPR \\ electron paramagnetic resonance \\ FCSD \\ Fundamental and Computational Sciences Directorate \\ MAS \\ magic-angle spinning \\ NMR \\ nuclear magnetic resonance \\ PNNL \\ Pacific Northwest National Laboratory
}




\section{Contents}

Executive Summary: Workshop Background, Purpose, and Organization ........................................ iii

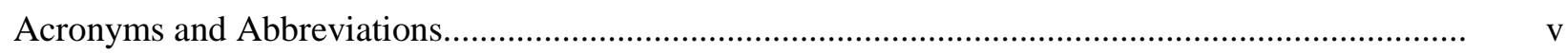

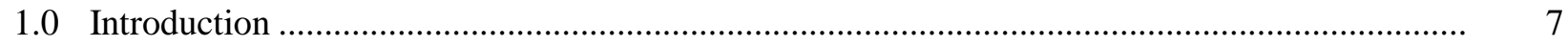

2.0 Summary of Findings and Recommendations .................................................................... 9

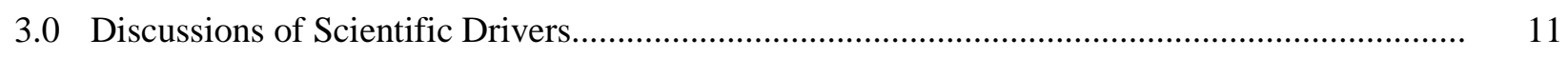

3.1 Breakout Session: Biological Sciences ......................................................................... 11

3.2 Breakout Session: Biogeochemical Sciences ................................................................... 13

3.3 Breakout Session: Materials Science ....................................................................... 15

3.4 Breakout Session: Catalysis Science ................................................................................... 16

4.0 Reaching the Next Generation of Magnetic Resonance ........................................................... 19

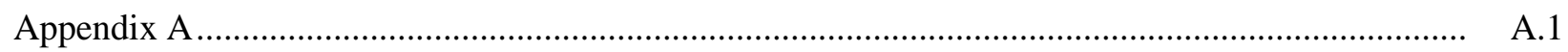

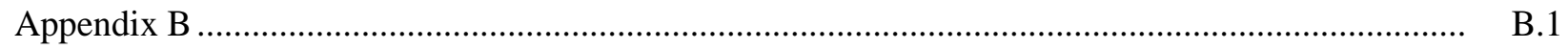

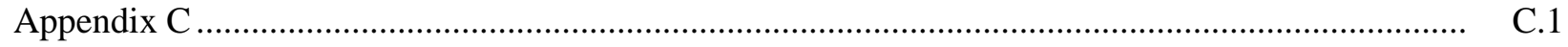




\subsection{Introduction}

Magnetic resonance is a powerful, high-resolution spectroscopic tool for the study of molecular structure and dynamics, and it has found wide adoption and use for studies of systems as wide-ranging as soil organic matter, proteins bound to vesicles or membranes, battery materials, catalysts, and biogeochemical interfaces. In modern nuclear magnetic resonance (NMR) studies, isotopic selectivity, or the ability to detect ${ }^{1} \mathrm{H},{ }^{13} \mathrm{C}$, and a range of other nuclei either separately or in concert, is combined with the dependence of NMR-derived parameters on local physical and chemical environments (measured through chemical shifts, relaxation times, spin couplings, etc.) to make NMR a widespread and preferred tool for advanced characterization of complex systems. Over the past decades, continued improvement in magnet technology has provided stable high-field strengths, attained fractions of parts-per-million homogeneity, and increased resolution and sensitivity by more than an order of magnitude. While selectivity and resolution are important success metrics for the performance of NMR as an analytic tool, NMR experiment sensitivity remains a limiting parameter for many applications. Non-incremental improvements in the sensitivity of magnetic resonance experiments, particularly NMR, will open broad, new sets of scientific problems and questions for exploration.

While NMR measurements are based on the spin angular momentum of atomic nuclei, the closely related technique of electron paramagnetic resonance (EPR) also provides in-depth details related to the local electronic and magnetic environments of unpaired electrons in chemical systems. Typical systems studied with EPR methods include inorganic transition metal complexes and organic/biological systems containing free radicals. Because EPR is based on the irradiation and detection of unpaired electron spins, this method is less pervasive than NMR for routine chemical analysis. Nevertheless, it provides distinct advantages in terms of selectivity and sensitivity. The polarization (related to the overall signal intensity) for unpaired electrons is approximately 660 times higher than that obtained from ${ }^{1} \mathrm{H}$ nuclei due to the smaller mass (therefore, higher gyromagnetic ratio) of the electron and is responsible for the higher sensitivity.

Showcase applications for both NMR and EPR magnetic resonance techniques have been well documented and broadly presented. As scientists and engineers expand the scope of problems they address using NMR and EPR techniques, a continuing issue that drives further development of magnetic resonance instrumentation and methodologies is the relatively low sensitivity of NMR compared to EPR. With respect to signal strength, NMR is a "weak" spectroscopy when compared to many other methods. Clear evidence also exists that coupling NMR and EPR techniques, which can occur through a variety of experimental designs, can provide critical access to additional information on chemical structure, dynamics, and reactivity. Indeed, a set of methodologies to take advantage of the stronger electron polarization within a magnetic field, while still providing the much more chemically relevant information from NMR, has been developed over the past half-century or more. One important and critical development (first demonstrated in the 1950s) is dynamic nuclear polarization (DNP). In a DNP experiment, electron spin polarization is efficiently transferred to nuclear spins via one or more mechanisms driven by microwave irradiation of the electron spins. Recent advances in gyrotron and related microwave instrumentation have resulted in a "renaissance" of DNP experimentation and development, and new applications have been demonstrated in the biological, energy, and environmental sciences. ${ }^{1}$ Regardless, recognized barriers to the future success of magnetic resonance techniques are: 1) further development of advanced instrumentation and 2) a sustainable venue for focused research efforts, including facile access for the general scientific community. For most research institutions, cost poses an additional and significant barrier, impeding magnetic resonance's progression.

\footnotetext{
${ }^{1}$ Griffin RG and TF Prisner. 2010. "High field dynamic nuclear polarization—-the renaissance.” Physical Chemistry Chemical Physics 12(22):5737-5740 and the accompanying articles in the special-themed issue of Physical Chemistry Chemical Physics on dynamic nuclear polarization.
} 



\subsection{Summary of Findings and Recommendations}

At the Environmental Molecular Sciences Laboratory (EMSL) workshop, attendees identified a large number of science drivers relevant to the goals and mission of the U.S. Department of Energy (DOE), Office of Science (SC). Problems especially relevant to DOE's Office of Biological and Environmental Research (BER) and Office of Basic Energy Sciences (BES) were discussed at length. A brief synopsis of the main discussion points and key science drivers identified during the four breakout discussions is included in Section 3.

To address these drivers with molecular specificity, increased sensitivity and selectivity for NMR experimentation is needed. The NMR technique poses a great advantage for researchers because it provides direct reporting on a variety of local environments by using chemical shift and quadrupolar parameters (the latter for nuclei with spin angular momentum quantum numbers of one or greater) to elucidate molecular structure and dynamics. Moreover, advances propelled by computational chemistry tools, such as the NWChem suite, an open-source set of computational tools developed at EMSL, allow for accurate and rapid comparison of calculated NMR parameters to experimental results.

As noted, DNP is emerging as the tool of choice for vast enhancement of NMR signals, exploiting the high spin polarization of nearby electrons. In addition to DNP capabilities, there is substantial interest in the design, development, procurement, and construction of a hybrid EPR and NMR system that could enable unparalleled detection of, for example, small quantities of metals in emerging valence and coordination states and allow accurate modeling of changes in structural and electronic environments using interleaved high-sensitivity NMR and EPR methods.

Discussions at the workshop identified an acute need for the development and deployment of DNP and coupled NMR/EPR capabilities at a venue that could provide the robust resources for addressing a large number of diverse scientific problems. While the EMSL facility provides world-class user capabilities for NMR and EPR, DNP development was curtailed after 2000 due to staff departures and focusing of resources on other applications, especially as technologies for high-frequency microwave irradiation remained under development. Meanwhile, a large "DNP gap” has emerged between laboratories in Europe and the United States. In the United States, instrumentation costs and the need for experts to further develop said instrumentation makes overcoming this gap a challenge. In light of the scientific drivers identified at the workshop and recent construction of a high-field (9.4 Tesla) EPR instrument at EMSL, coupled with a number of relevant strategic hires, EMSL has a tremendous opportunity to rapidly develop and deploy DNP capabilities. In particular, a partnership with Ames Laboratory (Iowa) and collaborations with academic partners (University of California, Santa Barbara; Montana State University; and University of Warwick [England]) and commercial vendors (Agilent, Bruker, and Bridge12 Technologies) were identified as possible directions that would accelerate development, installation, and exploitation of DNP or coupled NMR/EPR capabilities. 



\subsection{Discussions of Scientific Drivers}

The development of superior and transformative technology is driven by both concrete needs for analysis or description of complex systems and the desire to exceed limitations on previous technologies to extend their ability to approach new problems quickly and accurately. This workshop brought together thought leaders in a range of scientific disciplines to discuss drivers for improved technologies specific to magnetic resonance spectroscopy.

Four scientific breakout sessions were held as part of the workshop. Two breakout sessions ran in parallel during each full day of the event. Each breakout session was chaired by an EMSL or Pacific Northwest National Laboratory (PNNL) staff member and began with introductions followed by brief technical talks. These talks were presented by DOE-supported scientists and focused on cutting-edge and unresolved scientific questions in the fields of biological sciences, biogeochemical sciences, materials science, and catalysis science. After a brief period of questions and answers regarding the materials presented, broader scientific and technical discussions of the relevant scientific drivers in these fields commenced. Each discussion was mediated by the breakout session chair and featured speaker, who were asked to direct the discussions toward issues where increased sensitivity and selectivity in analytical analysis presented demanding needs for improved methodologies. Aided by a scribe, the leaders of each breakout session worked with participants to produce a list of top scientific drivers, and each session leader presented their list of drivers during a reporting session on the afternoon of the second day of the workshop. The following is a description of the main discussion topics from each session and a compilation of the main scientific drivers identified.

\subsection{Breakout Session: Biological Sciences}

External Participants: Christian Hilty, Cynthia Jenks, Melanie Rosay, David Singel, Jagadishwar Sirigiri, and Kent Thurber

PNNL Participants: Josh Adkins, John Cort, David Hoyt, Dave Koppenaal, Tom Squier, Eric Walter, Andrew Lipton, Steve Wiley, and Julie Wiley (scribe).

The Science Drivers in Biological Sciences breakout session was chaired by Dr. Andrew Lipton (PNNL/Fundamental and Computational Sciences Directorate [FCSD]) and featured a presentation by Dr. Steve Wiley (PNNL/EMSL) entitled, "NMR Applications in Biology in EMSL.” Dr. Wiley provided a broad overview of biologically focused research at EMSL and PNNL, which included a number of external collaborators. The main areas of interaction have involved protein structure determination, both general structural and dynamic protein-protein interaction studies, as well as metabolomics, a growing research area. In particular, Dr. Wiley noted that researchers increasingly are interested in targeted analyses; metabolite profiling; metabolic fingerprinting; and combined studies of metabolomics and metabonomics, an omics extension of metabolomics analyzing metabolic responses to chemicals, environmental changes, and diseases.

The topic of chemical complexity in global metabolomics analyses spurred significant discussion. An additional challenge included the emerging coupling of theory and experiment. It was noted that EMSL already plays an important part in the development of novel experiments, such as the deployment of mass spectrometry technologies and NMR to analyze metabolomics. However, in this field, the issue of sensitivity arises on a regular basis.

\section{Key Science Drivers}

- Metabolite identification in biological processes, focusing on kinetics and quantification. Metabolites are the end products of a variety of cellular processes and their high-throughput characterization and quantification can provide 
important insights into the functioning of living organisms. Further complex systemic behavior can be predicted or modeled from a "bottom-up" approach using accurate and comprehensive kinetic data. NMR is well suited to these measurements due to its non-invasive and non-destructive nature. However, for in vivo measurements, sensitivity could hamper the determination and study of kinetics if large numbers of signal averages are required. There is the possibility of using the longer spin-lattice relaxation times of nonprotonated nuclides $\left({ }^{15} \mathrm{~N}\right.$ or ${ }^{31} \mathrm{P}$, for example) to allow polarization enhancement in a DNP experiment. This polarization can be transferred back to protons for indirect detection methods. Complex mixtures also can be analyzed by coupling liquid chromatography (with or without additional mass spectrometric detection) with a flow NMR probe. While small sample sizes could limit experiments, enhancements via DNP would overcome this shortcoming.

- Breakdown of biomass. The deconstruction of biomass to useful energy feedstocks is of particular interest to DOE. Due to rising costs of fossil fuels and geopolitical concerns, a replacement is required, and generation of ethanol from lignocellulosic biomass represents a renewable and carbon-neutral alternative. Biofuels are naturally fermented as a result of microbes releasing sugars during breakdown of biomass. However, chemical dissolution of lignocellulose is difficult due to the cross-linked lignin walls. Characterization of the process of either fungal or microbial breakdown requires probing the interfacial region, which can be done non-invasively with NMR. Designing ionic liquids for costeffective dissolution of cellulose, lignin, and lignocellulose also requires characterization and quantitation that can be provided via NMR methods. However, as with any complex natural system, there are mixtures of components that will require multidimensional experiments to aid characterization. The main drawback for both tasks is sensitivity as isotopic labeling of biomass can be difficult. In contrast to current magnetic resonance capabilities (e.g., NMR), DNP could help avoid costly and complex isotopic labeling issues.

- Improved spectroscopy from metals (especially in proteins). Metal ions play an important role in bioinorganic chemistry. However, following their respective chemistries often is complicated because several relevant metal ions (such as $\mathrm{V}^{5+}, \mathrm{Cu}^{1+}, \mathrm{Zn}^{2+}, \mathrm{Na}^{+}, \mathrm{Ca}^{2+}$, and $\mathrm{Mg}^{2+}$ ) are not always amenable to conventional ultraviolet-visible (UV/Vis) or EPR spectroscopy. The critical nature of metals in biology requires a detailed understanding of the chemistry, structure, and bonding within these complexes and how, in turn, these manifestations alter the chemistry at the metal binding site. Toward that end, combining magnetic resonance parameters (chemical shift tensor and electric field gradients) with molecular theory provides key information. Difficulty arises when considering a metal site in a biological system, such as a protein. Consider a magnesium ion in a simple model compound such as magnesium acetate dihydrate: the metal is approximately $17 \%$ of the total mass. However, in the DNA repair protein polymerase $\beta$ (Pol $\beta-39 \mathrm{kDa}$ ), the percent $\mathrm{Mg}^{2+}$ is on the order of $0.13 \%$, or a dilution factor of $\sim 132$. Adding damaged DNA and including buffers/salts only makes this dilution greater. These types of systems are approachable using cryogenic NMR methods, but these techniques still require several days or more of signal averaging. If a multidimensional experiment (e.g., multiple-quantum magic-angle spinning, or MQMAS) or arrayed experiments (e.g., rotational echo double resonance, or REDOR) are desired, the experiment time becomes prohibitively long. These types of systems are a natural fit for DNP, which could decrease experiment time by an order of magnitude or more.

- Facile and rapid protein structure determination. Proteins are nature's molecular machinery and have typically evolved for particular purposes in a specialized environment. To understand how these molecular machines function, it is necessary to solve their three-dimensional structure. This can be accomplished non-destructively and under physiological conditions via NMR methods (either solution or solid state). However, to generate the restraints needed to solve a structure, several multidimensional experiments are performed, and each can require several days, depending on sample concentration (typically 0.1-3 mM). In cases where dilution cannot be avoided, such as membrane-bound or protein-protein complexes, experiment times can approach a full week. DNP enhancement would greatly facilitate these measurements to the point where the total multidimensional experiment time can be reduced to a single day rather than an entire week's worth of signal averaging. In extreme cases, enhanced sensitivity also would facilitate using gradients to divide a single sample and perform multidimensional spectroscopy in a single scan. 
- Whole-cell targeted spectroscopy (e.g., lighting up a particular organelle). Microbes are implicated as having solved (through evolution) several problems facing humans today, from breaking down biomass to sequestering heavy metals or even withstanding DNA damage due to ionizing radiation. To investigate how microbes accomplish these feats, researchers must characterize the cell either in its native community or through single-cell-type spectroscopies. Again, sensitivity becomes a major concern when considering the relative sizes of a cell, especially if the interest is a single organelle within the cell. However, much like fluorescent probes that target organelles, targeted radicals possibly could enhance the spectra from a particular organelle via DNP methods.

\subsection{Breakout Session: Biogeochemical Sciences}

External Participants: Song-I Han, Thorsten Maly, Melanie Rosay, David Singel, and Kent Thurber

PNNL Participants: Herman Cho, John Cort, Andy Felmy, Karl Mueller, and Nancy Washton

The Science Drivers in Biogeochemical Sciences breakout session was chaired by Dr. Nancy Washton (PNNL/EMSL) and featured a presentation by Dr. Andy Felmy (PNNL/FCSD) entitled, "Research Challenges in Geochemistry, Biogeochemistry, and Subsurface Science.” Dr. Felmy’s presentation began with an introduction to key problems addressed by DOE-funded research, including environmental sustainability, availability of water resources, environmental remediation, future energy technologies, geologic disposal, resource extraction, and strategic materials.

Examples were provided from research in areas such as environmental remediation, where scientists are interested in assessing the impact of waste, especially the interactions of waste with the surrounding environment; characterization of natural and microbial community interactions with these materials; and the chemical form of contaminants. Contaminants are especially difficult because they are usually found at low concentration, often are contained within complex phases, and may contain radioactive materials. Increased sensitivity is a key issue for spectroscopic interrogation of each of these concerns.

Another broad issue discussed was carbon cycling, in particular, geologic carbon sequestration. Current research focuses on predicting rates of mineral dissolution and formation of recalcitrant carbonate species. Here, one major challenge is determining reactive mineral phases and reaction products involved in these transformations. Additional challenges reside in the complexity of the mineral-water interface, the complexity of nanoparticle and colloid chemistry and physics, and following biogeochemical processes in extreme subsurface environments. Dr. Felmy also highlighted advances at EMSL in the exploration of mineral reactions and biogeochemistry with new in situ methods.

Dr. Washton, the session moderator, described her research on understanding the chemical species that control the dissolution of aluminosilicate surfaces, which is tied directly to wide-ranging topics in waste sequestration, transport of pollutants, abiotic and biotic oxidation-reduction chemistry, and catalysis. The discussions then progressed to major science drivers, where the sensitivity of novel analytical techniques (including magnetic resonance methods) could have a major impact.

\section{Key Science Drivers}

- Determination of complex solution phase speciation, including understanding of early nucleation events and untangling solution-state complexation (ligand selectivity). Biogeochemical studies focus on a number of fundamental reactions that occur in the environment, including aqueous dissolution and precipitation at interfaces. These reactions are important for waste removal, pollutant transport, and environmental electron transfer. In a number of previous studies at PNNL and elsewhere, scientists have studied the complex solution phase speciation of silicates 
as they attempt to formulate and modify the understanding of metal ion binding and phase nucleation and growth. In the simplest cases, many different chemical species can be identified and quantified, including those with different conformations or isomeric structures. Studies now must extend beyond simple silicate speciation to better evaluate metal ion complexation and the far-reaching area of nucleation and growth. However, the poor sensitivity of NMR spectroscopy for many nuclides in solution (especially ${ }^{29} \mathrm{Si}$ at environmentally relevant concentrations) severely limits new or expanded research efforts in this field. In each of these studies, combining computational tools with new techniques for increased sensitivity will be important in analyzing the results and defining the complexes that are present.

- Detailed descriptions of $\mathrm{CO}_{2}$ sequestration chemistry, including the identification of reactive mineral phases and reaction/transformation products. One proposed route to decreasing greenhouse gas emissions is pumping carbon dioxide that evolves from burning fossil fuels (such as coal) into subsurface aquifers, where gases and fluids may be stored under supercritical conditions. In these scenarios, $\mathrm{CO}_{2}$ that is disposed of as an anhydrous supercritical fluid can become water saturated during or after disposal in the subsurface. The less dense, more highly diffusive "wet" $\mathrm{CO}_{2}$ is highly reactive and is most likely to come into contact with the overlying caprock. Therefore, the study of the reactions of wet, supercritical $\mathrm{CO}_{2}\left(\mathrm{scCO}_{2}\right)$ with orthosilicate and phyllosilicate minerals is key to understanding mineral transformations that may occur in the subsurface, which are possibly quite different from the typical reactions observed under ambient conditions. Previous in situ and ex situ NMR studies of wet scCO $\mathrm{N}_{2}$ reactions with mineral surfaces have proven successful for identification of intermediate and product phases formed under conditions of high pressures beyond 90 atm. However, in a number of reactions, it has not been possible to identify small quantities of new silicate phases formed on the surfaces. Identification of these interfacial phases is necessary to define the mechanisms of the chemical reactions and better predict reaction rates.

- Structural and dynamical characterization of geochemical phase transformations and mineral-water interactions. As in the case of interfacial reactions of $\mathrm{scCO}_{2}$, many geochemical transformations occur at the interfaces of minerals with the aqueous solutions that contact them either temporarily or over long periods of time. The low surface areas of many geologically significant minerals, as well as the alteration of surface properties by either relatively large or small amounts of natural organic matter, makes molecular-level spectroscopic interrogation difficult. However, these studies are central to the development of geologic disposal for nuclear waste, energy extraction technologies (such as hydraulic fracturing), and pollutant transport. For example, ultra-low levels of actinide nanoparticles can drive the assessment of repository strategies for nuclear waste, yet there is no unified theory for colloid/nanoparticle transport in the subsurface. Indeed, at the mineral-water interface, there is an evolving research theme of moving from examination of static systems to evaluation of dynamics to examine long-term interfacial changes and reactivity. Increasing the sensitivity and time-resolution of characterization tools that are as powerful as NMR at determining molecular-level changes to structure and bonding are critical to these types of studies.

- Depth profiling for near-field, surface, and surface-bulk transitions. Chemical reactions at interfaces in the environment spread spatially across the interface from the far- to near-field as one approaches the surface from the solution side, on and through the surface's first atomic layer, then down into the solid's subsurface until the composition is again indistinguishable from the bulk. This total interfacial region depends intimately on the nature of the reaction; the activities of the reacting or diffusing chemical species; and other intrinsic properties, such as temperature and pressure. Depth- or position-sensitive probes of the structure and chemical dynamics in these systems would provide a missing description of the mechanisms and kinetics at these reacting interfaces that would be spatially dependent, providing a cross-sectional understanding of transport and reaction at the interfaces. Such studies are important for understanding, for example, transport and binding of radionuclides across interfaces of aluminoborosilicate glass proposed for vitrification of high-level nuclear waste. One proposed solution for obtaining 
position-sensitive NMR information is the utilization of spin labels (unpaired electrons) on variable-length linkers attached to surfaces for probing distance-dependent spin interactions.

\subsection{Breakout Session: Materials Science}

External Participants: Marc Caporini, Ulla Gro Nielsen, Song-I Han, John Hanna, Thorsten Maly, Marek Pruski, and Mark Smith

PNNL Participants: Don Baer, Greg Exarhos, Karl Mueller, Charity Plata (scribe), and Nancy Washton

The Science Drivers in Materials Science breakout session was chaired by Dr. Karl Mueller (PNNL/EMSL) and featured a presentation by Dr. Greg Exarhos (PNNL/FCSD) entitled, "Materials Science and Technology Drivers for Magnetic Resonance.” The presentation addressed broad areas in materials research, beginning with an overview of materials science and technology at PNNL. It was evident from the presentation that NMR has been a critical tool for understanding processes that help researchers develop technologies for diverse applications. At PNNL, DOE-BES serves as the primary funding source for materials research and has provided its strategic directions and goals by way of a "Five Challenges” report. ${ }^{2}$ For example, in the Materials Genome Initiative, a major goal is to double the speed of discovery, development, and manufacture of materials. Another emerging challenge is in the area of mesoscale science. Dr. Exarhos described six general materials research themes at PNNL: 1) interfacial modification, 2) self-assembly scalable synthesis, 3) defect-driven properties, 4) structural transformation, 5) environmental interactions, and 6) transport phenomena. In each area, he presented examples where magnetic resonance technology could, in principle, provide outstanding information if the technique’s sensitivity was increased.

Dr. Mueller also provided a brief overview of his research using solid-state NMR to study the interfaces of oxide materials. In these systems, the issues of selectivity, sensitivity, and resolution are clearly limiting for many systems. Examples were presented of research addressing the coupling of polymers and binders with glass and other complex oxide surfaces. Model compounds, such as ${ }^{13} \mathrm{C}$-enriched ethanol or acetic acid, can be used to investigate surface reactivity with different sets of reactions based on varied oxide surfaces. If the sensitivity could be enhanced by even an order of magnitude, Dr. Mueller proposed that selective analysis of local reactivity would be possible. Truly forward-looking applications would include analyzing very-low-surface-area materials and in situ flow at elevated temperatures.

\section{Key Science Drivers}

- Characterization of spatially resolved template self-assembly mechanisms as a prelude to the development of scalable synthesis processing routes. Current research areas of great interest to DOE include hierarchical growth and controlled self-assembly (including the dynamics of assembly and the evolution of porosity in hierarchical systems). The control and specific tailoring of porosity, moving from the nanoscale through the microscale and to the mesoscale, impacts major materials systems for applications including energy storage, sequestration, and catalysis. The goal of DOE-driven research programs at PNNL and elsewhere is to obtain fundamental understandings of synthetic approaches to control structures and properties of nanostructured systems, as well as couple and drive these understandings using iterations with both advanced computational tools and transformational experimental tools to observe and control atomic-scale processes. Increased sensitivity for spectroscopic analyses such as NMR is essential for studying these tailored systems, providing unparalleled molecular-level details and reporting on both complex and specific structural information and the kinetics of complex assemblies formation.

\footnotetext{
${ }^{2}$ BESAC - Basic Energy Sciences Advisory Committee. 2007. "Directing Matter and Energy: Five Challenges for Science and the Imagination.” U.S. Department of Energy, Washington D.C.
} 
- Understanding of defect-driven properties. Impurity incorporation in single materials has been used for decades to drive the properties of materials for applications of wide interest and importance. Examples include ion conductivity in electrodes and membranes with applications for energy storage (batteries), electron conductivity in transparent conducting oxides (including aluminum-doped zinc oxides), and polarons in conducting polymers and transition metal spinel oxides. The migration of atoms across oxide-oxide interfaces, for example, as occurs in induced conductivity from thermodynamic stability at the $\mathrm{LaAlO}_{3} / \mathrm{SrTiO}_{3}$ interface, also is increasingly important. The mechanisms of strengthening and hardening of materials also is affected by defect incorporation, as well as grain size, control, and strain. In all of these fundamental applications, the interplay of structural, compositional, mechanical, and chemical effects play off one another to provide superior materials for advanced applications. Understanding the fundamental structural details of defect and impurity incorporation is difficult due to the low number density of sites in these materials. Therefore, sensitive spectroscopies, coupled with advanced computational tools, are critical for the rational design of new and improved materials.

- Interfacial modification and the study of defect formation and incorporation. A number of research areas and technical applications stress the utility of interfacial modification, including the development of mirrors based upon multilayer dielectric stacks. The challenge in a number of these applications is the structural and chemical characterization of the interphase region, where two materials are in contact. In particular, buried interfaces pose a particular characterization challenge. The low number of sites at the interface, or in the interphase, in comparison to those in the bulk of the system increases the difficulty of measurements to determine the properties of the modified regions. In this case, the placement of reporter spins (radicals or highly polarizable nuclei) with characteristics amenable to advanced magnetic resonance methods would provide novel analyses.

- Shortening of data acquisition times to study transport phenomena. The migration and transport of atoms, molecules, and ions underlie many important chemistries that operate in materials, ranging from glasses to polymers, as well as many crystalline materials. One of the most important applications of charge transport is, of course, battery systems, where the transport of charge may be used to store and then release energy via electron flow through an external circuit. While a number of in situ technologies have been developed to characterize battery components, one limiting factor is the time required for each measurement compared to the system's typical charge/discharge cycle. Increased measurement sensitivity comes with a time savings roughly proportional to the square of the gain in sensitivity. As such, even incremental increases in signal strength will shorten observation times and lead to new discoveries in areas related to transport phenomena.

\subsection{Breakout Session: Catalysis Science}

External Participants: Marc Caporini, John Hanna, Cynthia Jenks, Takeshi Kobayashi, Olivier Lafon, Marek Pruski, Jagadishwar Sirigiri, and Mark Smith

PNNL Participants: Johannes Lercher, Andrew Lipton, Chuck Peden, Charity Plata (scribe), Jesse Sears, Eric Walter, and Hongfei Wang

The Science Drivers in Catalysis Sciences breakout session was chaired by Dr. Marek Pruski (Ames Laboratory) and featured a presentation by Dr. Chuck Peden (PNNL/FCSD) entitled, “Catalysis Studies with NMR Spectroscopy.” Dr. Pruski began the session with an introduction, explaining the goals and challenges in catalysis science that might be resolved using advanced NMR methods. Following this introduction, Dr. Peden presented an overview of scientific areas where many researchers have needs not easily met by current magnetic resonance techniques. He showcased a number of current research areas of interest to DOE and the nation, including security and environmental concerns. For example, DOE is interested in how future feedstocks for fuels, especially biomass, can be transformed into useful fuels. Dr. Peden 
also discussed the broader vision at the technology scale, from discovery research to technology maturation and deployment. Ultimately, his view is that to understand catalytic chemistry, scientists must begin to unravel catalysts at the atomic level. Then, the next challenge is to couple the structure with catalyst reactivity.

Focusing on NMR spectroscopy, Dr. Peden noted that many nuclei ubiquitous in catalytic systems often are difficult to study. Demonstrations of superior results have used major advances resulting from high magnetic field strengths and high magic-angle spinning (MAS) rotational speeds. However, when considering solid catalyst materials, the need for MAS makes it difficult to perform experiments under in situ or operando conditions. Sensitivity, in general, is a real issue for NMR, which embodied and became the focus of this workshop session.

As a comment from the "non-expert” in the field of magnetic resonance, Dr. Peden also noted that NMR spectroscopy is particularly difficult to understand. Therefore, advancement of new technologies works well within a user facility such as EMSL, where scientists and experts in advanced analysis and spectroscopy are available to assist and collaborate with users.

Furthermore, Dr. Peden explained how most catalytic conversions take place on oxide-supported surfaces of catalytic particles and how the abundance of quadrupolar spins in these systems makes quantification difficult. However, ${ }^{27} \mathrm{Al}$ spectra obtained at 21.1 Tesla (900 $\mathrm{MHz}$ for ${ }^{1} \mathrm{H}$ ) were used to follow the conversion of five-coordinate aluminum species to six-coordinate species in a catalytic system as a function of loading Pt on to an alumina surface, which could not have been accomplished without the improved resolution and sensitivity afforded by working at higher fields. Other examples and discussions focused on why operando analysis of catalysts using magnetic resonance is so important, as well as the power of mining information from multiple spectroscopies. These types of experiments are important for following the mechanism of the chemical reaction itself by monitoring peaks from reactants and products and improve the ability to study the catalyst and its changes while a catalytic reaction is running.

\section{Key Science Drivers}

- Realistic quantitative and qualitative descriptions of all reactive sites in a sample, including the interactions between these sites and reactants, as well as coupling of structural information with reaction kinetics. While it is well recognized that solid-state NMR spectroscopy is capable of providing detailed atomic-scale information about the structure and functions of heterogeneous catalytic systems, inherently low sensitivity limits conventional solidstate NMR's ability to characterize catalytic surfaces containing nuclei with low gyromagnetic ratios and/or low natural abundance. However, recent studies have demonstrated that DNP-induced "hyperpolarization” of nuclear spins can be achieved by impregnating porous catalysts with appropriate solutions of biradical polarizing agents. This results in a dramatic increase in sensitivity, corresponding to a thousand-fold improvement in time performance, which enables studies of smaller concentrations of catalytic groups, reactants, and intermediates or respectively smaller catalytic surfaces. Similarly, using DNP-enhanced NMR measurements will afford time-resolved studies of catalytic processes.

- Advanced characterization of tethered catalysts. Detailed information about the structure of complex catalytic systems, especially tethered catalyst systems, requires characterization of first- and second-coordination sphere catalytic environments, studies of cooperative catalytic phenomena, and optimization of structure-activity relationships to move catalysis science forward. All of these can be provided by homo- and hetero-nuclear correlation methods, which separate the resonances of interacting spins in more than one dimension. However, in these complex systems and experiments, sensitivity concerns again move to the forefront. Such measurements require acquisition of multiple one-dimensional spectra. Thus, they often are impossible in catalytic systems featuring small quantities of studied nuclei. DNP-based methods will enable these characterizations by enhancing the ${ }^{1} \mathrm{H}$ polarization and subsequent transfer to other spins, such as ${ }^{13} \mathrm{C},{ }^{15} \mathrm{~N}$, and ${ }^{29} \mathrm{Si}$. The challenges will be in improving fast MAS 
capabilities at low temperatures, usually required for DNP (including better control of frictional heating), and development of better biradicals and solvent-free approaches to eliminate perturbations due to solvents. The resulting methodology will enable characterization well beyond the current limits of sensitivity, opening up a range of systems to novel examination and improvement.

- Sensitivity enhancement for small catalytically active phases and improved interrogation of metal sites. A group of nuclei that include ${ }^{25} \mathrm{Mg},{ }^{47 / 49} \mathrm{Ti},{ }^{43} \mathrm{Ca},{ }^{47 / 49} \mathrm{Ti}$, ${ }^{55} \mathrm{Mn},{ }^{78} \mathrm{Sr},{ }^{91} \mathrm{Zr},{ }^{95} \mathrm{Mo},{ }^{113} \mathrm{Cd}$, and ${ }^{119} \mathrm{Sn}$ are located both in catalytic support materials and within functional catalytic groups themselves. In many (if not most) real-world catalytic systems, NMR studies involving all of these nuclei remain beyond current detection limits due to low sensitivity. Future DNP measurements will enable studies of catalytically important systems where these nuclei are present. Interestingly, in line with many emerging developments in the field, samples where ${ }^{1} \mathrm{H}-{ }^{1} \mathrm{H}$ spin diffusion cannot be used to transfer nuclear magnetization may be studied using so-called “direct DNP," which utilizes doped or endogenous paramagnetic centers. 


\subsection{Reaching the Next Generation of Magnetic Resonance}

This workshop set the stage for establishing future directions in the development and deployment of next-generation magnetic resonance spectroscopies. The importance of the instrumentation and applications were brought to the forefront, and a collection of experts and users of magnetic resonance techniques worked to identify crucial scientific gaps and needs that could be addressed by a combination of improved sensitivity, selectivity, and resolution.

Three specific actions were recommended to address the needs and desired capabilities identified by scientists in areas that fall within the scientific goals and missions of BER and BES. For the initial action, a future workshop should be considered with the dual purposes of focusing on novel scientific drivers, with a broader range of participants, and addressing the technical challenges for magnet and irradiation technologies for the proposed move to higher magnetic field strengths. For scientists unfamiliar with current, high-value use of novel instrumentation and techniques, a tutorial session on modern magnetic resonance methods would be presented, incorporating an introduction to proposed future developments based on the results of this current workshop. This future workshop would be convened as a coordinated effort with proposed partner organizations. Gains in sensitivity and knowledge from a move to higher fields can only be realized with a concerted push forward in technologies, and experts from academia, government laboratories, and private industry will need to engage one another to achieve progress. As a result, a series of in-depth discussions should be held with envisioned outcomes that include the formation of working groups for the production of sciencebased technical specifications for new capabilities.

The second action is to establish, as quickly as possible, capabilities for DNP in the United States to address questions relevant to DOE missions. This equipment should be made available to external researchers. The recommended path forward should focus on the acquisition of an initial DNP instrument based on existing technology and commercially available products. Possible strategies include the purchase of a DNP system from a commercial vendor (approximate direct cost: \$1.5 million to \$2 million); purchasing partial equipment from a commercial vendor while using existing magnet(s), reducing the cost by up to 50\%; or utilizing new or existing magnets while engaging in a partnership for production/testing/deployment of gyrotron technologies. The latter avenue would present lower capital costs but would also require a longer development time and increase risk.

In the longer term (a three- to six-year time frame), EMSL is strongly positioned to lead the development of a unique "next-generation magnetic resonance" spectrometer. Further investigation of the scientific drivers and technical feasibility of such an undertaking are warranted, including additional consultation with experts and stakeholders. A next-generation spectrometer would couple ultra-high-field magnets—up to 28.1 Tesla, or $1.2 \mathrm{GHz}$ for ${ }^{1} \mathrm{H}$ NMR resonance frequencies—-with developing technology in the terahertz region of the EPR spectrum (provided by commercial enterprises) and leverage established EMSL excellence in building unique magnetic resonance probes. In this case, novel probes would be for combining high-resolution NMR, EPR, and DNP—all within the highest-field magnetic resonance spectrometer possible. Development would occur as a Major Item of Equipment (MIE) acquisition, wherein EMSL proposes to develop and acquire a new, cutting-edge magnetic resonance capability for simultaneous electron- and nuclear-spin magnetic resonance measurements with orders of magnitude higher sensitivity. Coupling these measurements with results from computational chemistry on a near-real-time basis would be achieved through a close link between EMSL's magnetic resonance capabilities and NWChem platform developers and users. A number of technical options for acquiring a next-generation magnetic resonance capability should be evaluated in close collaboration with academic partners and technology companies, as well as other DOE magnetic resonance experts, such as Dr. Pruski and co-workers at Ames Laboratory. 



\section{Appendix A}





\title{
Appendix A: Summaries/Lecture Abstracts
}

\section{Chemical and Biochemical Reactions Investigated by Dynamic Nuclear Polarization}

\author{
Dr. Christian Hilty \\ Texas A\&M University, Department of Chemistry, College Station, TX 77843, USA; Phone: 979-862-3099; \\ E-mail: chilty@chem.tamu.edu
}

Solid-to-liquid state dynamic nuclear polarization (DNP) affords a sensitivity gain sufficient to enable single-scan acquisition of NMR spectra at low concentration, of insensitive nuclei, or of nuclei with low natural abundance.

Combined with a rapid injection and mixing system, it becomes possible to obtain time-resolved data characterizing dynamic processes far from equilibrium. Kinetics and intermediates can be observed in various types of reactions, including enzyme-catalyzed reactions of small-molecule substrates, fast organic reactions, and polymerization reactions. By observing nuclei with large chemical shift dispersion, such as ${ }^{13} \mathrm{C}$, even closely related reactants and products can be distinguished, such as different anomers of sugars. Quantitative analysis of the signal evolution additionally reveals information on relaxation rates, which are dependent on structural and dynamic properties of the molecular species involved. Interactions of small molecule ligands with proteins can be investigated by observing the effect of binding on the ligand signals, which allows the determination of dissociation constants, and can also be used to determine competitive binding of two ligands to a binding pocket. Further, protein resonances can be enhanced by transfer of magnetization through nuclear Overhauser effect from hyperpolarized ligands. Finally, data will be presented showing that substantial signal enhancements can be obtained when polarizing polypeptides directly, which opens a new avenue for the study of protein folding. 


\title{
Dynamic Nuclear Polarization at High Magnetic Field: How Can Electrons Align Nuclei?
}

\author{
Olivier Lafon \\ UCCS, UMR CNRS 8181, Université of Lille Nord de France, F-59652 Villeneuve d'Ascq, France \\ E-mail: olivier.lafon@ensc-lille.fr
}

Low sensitivity is an important limitation of NMR spectroscopy, microscopy, and imaging. This low sensitivity precludes the use of NMR techniques to observe diluted species (reaction intermediates, defects, etc.), low volume samples (isotopically labeled biomolecules, lab-on-a-chip studies, etc.), interfaces (catalysts, hybrid materials, etc.), and nuclei displaying small magnetic moments.

In this context, Dynamic Nuclear Polarization (DNP) represents a broadly applicable technique to enhance the sensitivity of solution and solid-state NMR spectroscopy, as well as MRI. In DNP, the polarization from unpaired electrons is transferred to the detected nuclei by microwave irradiation at or near the electron paramagnetic resonance (EPR) frequency. Originally introduced at low magnetic field $^{1,2}$, DNP was then applied under magic-angle spinning (MAS) and at high-magnetic field to combine sensitivity and high-resolution. ${ }^{3-7}$ The gain in sensitivity offered by high-field DNP has been applied for the study of membrane proteins ${ }^{8}$ or porous materials. ${ }^{9-10}$

Here, we first present the different polarization transfer mechanisms under continuous microwave irradiation. We discuss the paramagnetic species, which can be used as polarization agent, and how they can be incorporated into the investigated sample. We describe the instrumentation required for high-field DNP. Finally, we discuss some recent applications.

\section{References}

1. A. W. Overhauser, Phys. Rev., 92, 411-415 (1953).

2. T. R. Carver et al., Phys. Rev., 92, 212-213 (1953).

3. R. Wind et al., Prog. Nucl. Magn. Reson. Spectrosc., 17, 33-67 (1985).

4. L. R. Becerra et al., Phys. Rev. Lett., 71, 3561-3564 (1993).

5. D. A. Hall et al., Science, 276, 930-932 (1997).

6. T. Maly et al., J. Chem. Phys., 128, 052211 (2008).

7. A. B. Barnes et al., Appl. Magn. Reson., 34, 237-263 (2008).

8. V. S. Bajaj et al., Proc. Natl. Acad. Sci., U.S.A. 105, $9244-9249$ (2009).

9. A. Lesage et al., J. Am. Chem. Soc., 132, 15459-1561 (2010).

10. O. Lafon et al., Angew. Chem. Int. Ed., 50, 8367-8370 (2011).

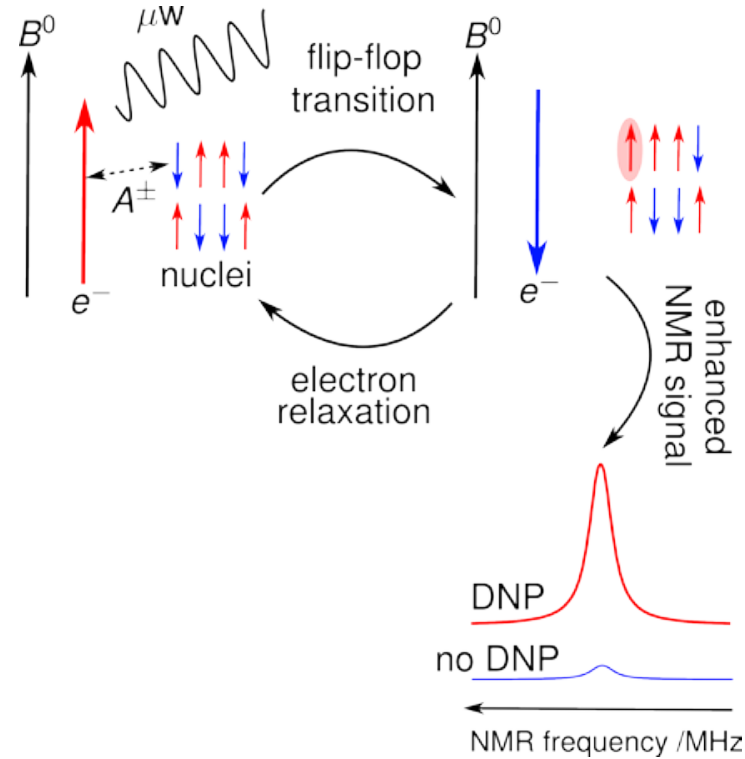

Figure: Principle of DNP using solid-effect as a polarization transfer mechanism. The non-secular terms of the electron-nucleus (hyperfine) interaction are denoted $A^{ \pm}$. 


\title{
Opportunities of DNP for the Study of Interactions and Interfaces in Solutions and Solids
}

\author{
Professor Song-I Han \\ Department of Chemistry and Biochemistry, University of California, Santa Barbara, CA 93106-9510 \\ E-mail: songi@chem.ucsb.edu
}

I will present approaches to pushing the sensitivity limits of nuclear magnetic resonance (NMR) spectroscopy for the study of materials, macromolecular complexes, and biological interactions. Particular emphasis is on the interrogation of interfaces and local structures through locally amplified NMR spectroscopy and solid materials, as well as biological systems in solution state. The study of local features at the nanometer and sub-nanometer scale is achieved through the use of strategic or intrinsic electron spin probes and by employing orders of magnitudes of signal enhancements obtained through the polarization transfer from the electron spin probes to the surrounding nuclear spins, a process termed “dynamic nuclear polarization” (DNP).

I will present instrument and method development efforts, as well as case studies of the microstructure of multidomain polymer solids and the site-specific study of the local hydration dynamics of biomolecular surfaces in solution systems. 


\title{
Recent DNP Work at Warwick - Investigating Factors Determining DNP Signal Enhancement from Liquids at 94 GHz and MAS DNP NMR Using a Quasioptical Microwave Transmission Design
}

\author{
Mark E. Smith \\ Department of Physics, University of Warwick, UK, CV4 7AL \\ E-mail: M.E.Smith.1@warwick.ac.uk
}

DNP-enhanced ${ }^{1} \mathrm{H}$ NMR at $143 \mathrm{MHz}$ is investigated in toluene using electron irradiation at $94 \mathrm{GHz}$ of the TEMPOL polarisation agent. The maximum enhancement for the ring protons increases from -16 for a 5-mM TEMPOL solution to -50 for a 20 -mM solution at a microwave power of $\sim 480 \mathrm{~mW}$. The temperature dependence of the enhancement, the NMR relaxation rates, and the EPR spectrum of TEMPOL provide information on the dynamics of the system. For water protons at 3.4 T using TEMPOL as a polarising agent, it is shown that for high radical concentrations ( 100 mM), the leakage factor is approximately 1 and, provided sufficient microwave power is available, the saturation factor is also approximately 1 . In this situation, the DNP enhancement is solely a product of the ratio of the electron and nuclear gyromagnetic ratios and the coupling factor enabling the latter to be directly determined. The temperature variation of $T_{1 I}$ allows accurate modelling of the signal enhancement behaviour, which varies linearly with temperature over a certain temperature range. Large DNP enhancements $(>-100)$ are reported at high temperatures, but it is also shown that, through adjustment of the microwave power and irradiation time, significant enhancements (e.g., -40) can be achieved whilst maintaining the sample temperature at $40^{\circ} \mathrm{C}$. A solid-state DNP-enhanced MAS NMR spectrometer, designed to operate at both 6.7 and $14.1 \mathrm{~T}$, will be described in detail that uses quasi-optics for microwave transmission. Initial data for $6.7 \mathrm{~T}$ and $\sim 1 \mathrm{~W}$ microwave power have been shown to produce enhancements of 60 for a frozen urea solution $\left({ }^{1} \mathrm{H}-{ }^{13} \mathrm{C} \mathrm{CP}\right), 16$ for a protein embedded in a membrane $\left({ }^{1} \mathrm{H}-{ }^{13} \mathrm{C} \mathrm{CP}\right)$, and 22 for a frozen glycine solution $\left({ }^{1} \mathrm{H}-{ }^{15} \mathrm{~N} \mathrm{CP}\right)$.

\section{Acknowledgement}

There were many contributors to this project, which was supported by EPSRC (UK) Basic Technology grants EP/D045967, EP/D047730, and EP/D047005. We thank Doty Scientific Inc. for their cooperation in the development of the MAS NMR probe, Adrian Lovejoy and David Greenshields of the Electronics Workshop in the Physics Department at the University of Warwick, and the NMR Magnet Group in The Magnet Technology Centre at Agilent Technologies UK Ltd. 


\title{
Starburst Dendrimers with Tailored Spin Labeling for DNP at High Fields
}

\author{
David J. Singel \\ Department of Chemistry and Biochemistry, Montana State University, Bozeman MT 59717 \\ E-mail: dsingel@chemistry.montana.edu
}

We are exploring the magnetic properties of macromolecular templates lavishly loaded with nitroxide spin labels. One objective of these studies is to develop electron spin systems with properties optimized for DNP (dynamical nuclear polarization) via thermal mixing - a mechanism conducive to efficient polarization transfer at high magnetic fields and fundamentally dependent on the dipolar spectrum of the electron-spin system. Over the past several years, the Singel and Cloninger groups at MSU have been collaborating on the synthesis and characterization of nitroxide-functionalized starburst dendrimers with the long-range view of developing the means to tailor electron spin dipolar spectra for application as polarization sources in DNP.

In PAMAM (poly amino-amido) dendrimers of generation 2 through 6 and with loadings varying from 0-100\%, we find dipolar spectra that are typically Lorentzian in shape (with broad-winged super-Lorentzian line shapes favored at highloading and low-generation numbers) and have with widths (full-widths at half-maximum) ranging to over $20 \mathrm{G}$, and with greater widths (and greater spectral densities at the desired nuclear Larmor frequency offsets) at greater loadings. Overall, we have secured a library of spin probes with a wide variation in dipolar spectra. Previous DNP work with mono- and biradical systems may be viewed as sampling the low loading limit of large dendrimers. Our spin probe library will enable the systematic variation of electron dipolar spectra in efforts to achieve optimal DNP enhancements. 


\section{High-field ${ }^{29} \mathrm{Si},{ }^{13} \mathrm{C}$, and ${ }^{27} \mathrm{Al}$ Dynamic Nuclear Polarization of Porous Materials and Nanoparticles}

Olivier Lafon ${ }^{1}$, Melanie Rosay ${ }^{2}$, Fabien Aussenac ${ }^{3}$, Aany Sofia Lilly Thankamony ${ }^{1}$, Xingyu Lu ${ }^{1}$, Julien Trébosc $^{1}$, Nadia Touati ${ }^{4}$, Alain Moissette ${ }^{4}$, Hervé Vezin ${ }^{4}$, and Jean-Paul Amoureux ${ }^{1}$

${ }^{1}$ UCCS, UMR CNRS 8181, Université of Lille Nord de France, F-59652 Villeneuve d’Ascq, France; ${ }^{2}$ Bruker Biospin Corporation, 15 , Fortune Drive, Billerica, MA 01821, USA; ${ }^{3}$ Bruker Biospin SA, 67166 Wissembourg, France; ${ }^{4}$ LASIR, UMR CNRS 8516, Université of Lille Nord de France, F-59652 Villeneuve d'Ascq, France

E-mail: olivier.lafon@esnc-lille.fr

Solid-state NMR is an irreplaceable technique for probing the atomic-scale structure and dynamics in inorganic materials. However, its applicability is plagued by a lack of sensitivity, which prevents the observation of interfaces; diluted species (defects, dopants); or isotopes, such as ${ }^{29} \mathrm{Si}$, with low receptivity or long nuclear longitudinal relaxation time. Dynamic Nuclear Polarization (DNP) is a promising method to enhance the NMR signals by one or two order of magnitude. This technique is based on the microwave-driven transfer of polarization from unpaired electrons to the nuclear spins. However, DNP at high field has been mainly demonstrated for ${ }^{13} \mathrm{C}$ and ${ }^{15} \mathrm{~N}$ nuclei of organic and biological compounds in frozen solution.

The extension of high-field MAS DNP to inorganic materials faces the issue of polarization distribution throughout non-protonated solids, in which ${ }^{1} \mathrm{H}-{ }^{1} \mathrm{H}$ spin diffusion is precluded. We show here how direct ${ }^{29} \mathrm{Si}$ DNP allows circumventing this limitation in the case of siliceous materials with applications in photonics, nanotechnology, and catalysis. ${ }^{1}$ Signal enhancement up to 30 were measured by direct ${ }^{29} \mathrm{Si}$ DNP. Furthermore, as shown in the figure, direct ${ }^{29} \mathrm{Si} \mathrm{DNP}$ and indirect ${ }^{29} \mathrm{Si}$ DNP via ${ }^{1} \mathrm{H}$ are complementary. ${ }^{2}$ The former enhances both the surface and subsurface ${ }^{29} \mathrm{Si}$ signals, while the latter enhances the surface ${ }^{29} \mathrm{Si}$ signals selectively. Furthermore, other recent applications of high-field DNP for porous solids and nanoparticles will be presented.

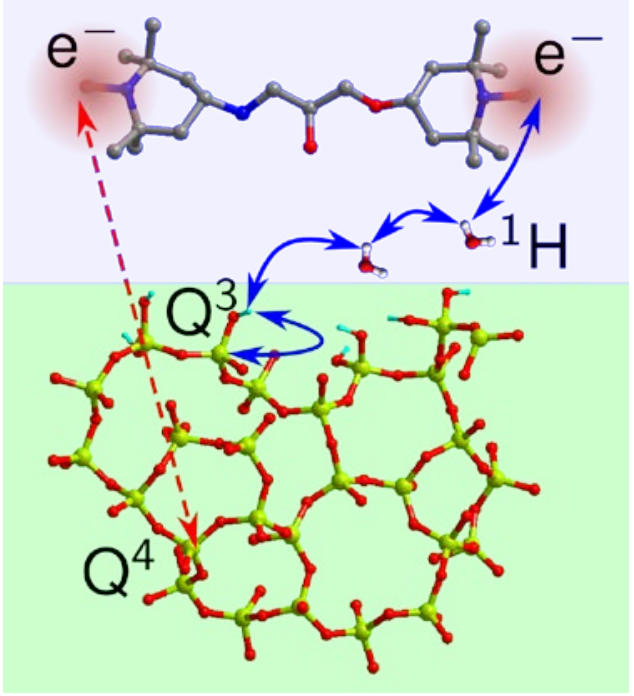

Figure: Direct ${ }^{29}$ Si DNP (dashed arrow) and indirect ${ }^{29} \mathrm{Si} \mathrm{DNP}$

(blue arrows).

\section{References}

1. O. Lafon et al., Angew. Chem. Int. Ed., 50, 8367-8370 (2011).

2. M. Lelli et al., J. Am. Chem. Soc., 133, 2104-2107 (2011). 


\title{
DNP with MAS at 9 Tesla and $25 \mathrm{~K}$ : Theoretical and Experimental
}

\author{
Kent R. Thurber, Wai-Ming Yau, Alexey Potapov, and Robert Tycko \\ Laboratory of Chemical Physics, National Institute of Diabetes and Digestive and Kidney Diseases, National Institutes of Health, \\ Bethesda, MD 20892-0520 \\ E-mail: thurberk@niddk.nih.gov
}

Dynamical Nuclear Polarization (DNP) is a technique for large sensitivity enhancements (10-100 times) of solid-state NMR. The increased sensitivity can enable solid-state NMR studies of a variety of dilute biomolecular systems, such as protein-folding intermediates, intermediates on the pathway to amyloid formation, and hormone/receptor complexes. We have demonstrated that significant DNP enhancements can be achieved through the cross-effect DNP mechanism with low microwave power $(30 \mathrm{~mW})$ if the sample temperature is low $(<30 \mathrm{~K})$. In this talk, I will review published results for non-spinning samples and present ongoing work in which we are developing a magic-angle spinning DNP system for 9.4 $\mathrm{T}$ and 20-25 K and investigating theoretical aspects of cross-effect DNP under MAS. Experimentally, our DNP-MAS probe uses 4-mm-diameter zirconia rotors and is based on our previous work on MAS at low temperature using helium to cool the sample — while still using nitrogen gas for the bearings and spinning gas. I will also present theoretical calculations of cross-effect DNP with MAS using radicals with a large electron g-anisotropy, as is the case for the Tempo nitroxides used in our experiments. If 1/T1e is significant relative to the MAS speed, the cross-effect can be viewed as two separate events: 1) the saturation of one electron spin by the microwaves and 2) the three-spin interaction between two electrons and one nucleus, which can transfer polarization to the nuclear spin if the electrons have a polarization difference. 


\title{
Extending Applications of Solid-State MAS-DNP NMR to New Systems and Nuclei
}

\author{
Marc A. Caporini* \\ Institut des Sciences et Ingénierie Chimiques, Ecole Polytechnique Fédérale de Lausanne, EPFL, Batochime, 1015 Lausanne, \\ Switzerland (*Current Affiliation: Bruker BioSpin Corporation, Billerica, MA 01821 USA) \\ E-mail: marc.caporini@gmail.com
}

Recent developments of high-field dynamic nuclear polarization (DNP) in combination with magic angle spinning (MAS) have greatly expanded the capabilities of solid-state NMR by increasing the sensitivity of experiments by a few orders of magnitude. Through the pioneering work of Griffin and coworkers ${ }^{1}$ in developing the technique and the commercialization of the hardware by Bruker ${ }^{2}$, it is now fairly routine to perform in situ MAS-DNP experiments on solid samples containing radicals at temperatures around $100 \mathrm{~K}$ while using a gyrotron to irradiate the radicals for DNP. The initial success of these experiments was largely demonstrated on biological systems ${ }^{1-6}$ focusing on ${ }^{1} \mathrm{H},{ }^{13} \mathrm{C}$, and ${ }^{15} \mathrm{~N} N M R$. Currently, we are extending the applicability of this technique to materials ${ }^{7-8}$, particularly functionalized silica and alumina surfaces using ${ }^{29} \mathrm{Si}$ and ${ }^{27} \mathrm{Al}$ NMR in addition to ${ }^{13} \mathrm{C}$ and ${ }^{15} \mathrm{~N}$. In many cases, DNP can provide the sensitivity boost necessary for NMR structural characterization of organic functional groups on surfaces in low concentrations that would otherwise not be possible.

\section{References}

1. L. R. Becerra, G. J. Gerfen, R. J. Temkin, D. J. Singel and R. G. Griffin, Phys. Rev. Lett., 1993, 71, 3561-3564.

2. M. Rosay, L. Tometich, S. Pawsey, R. Bader, R. Schauwecker, M. Blank, P.M. Borchard, S.R. Cauffman, K.L. Felch, R.T. Weber, R.J. Temkin, R.G. Griffin and W.E. Maas, Phys. Chem. Chem. Phys., 2010, 12, 5850-5860.

3. V. S. Bajaj, M. L. Mak-Jurkauskas, M. Belenky, J. Herzfeld and R. G. Griffin, Proc. Natl. Acad. Sci. U. S. A., 2009, 106, 92449249.

4. P. C. A. van der Wel, K.-N. Hu, J. Lewandowski and R. G. Griffin, J. Am. Chem. Soc., 2006, 128, 10840-10846.

5. G. T. Debelouchina, M. J. Bayro, P. C. A. van der Wel, M. A. Caporini, A. B. Barnes, M. Rosay, W. E. Maas and R. G. Griffin, Phys. Chem. Chem. Phys., 2010, 12, 5911-5919.

6. C. Song, K.-N. Hu, C.-G. Joo,-T. M. Swager and R. G. Griffin, J. Am. Chem. Soc., 2006, 128, $11385-11390$.

7. A. Lesage, M. Lelli, D. Gajan, M. A. Caporini, V. Vitzthum, P. Miéville, J. Alauzun, A. Roussey, C. Thieuleux, A. Mehdi, G. Bodenhausen, C. Copéret and L. Emsley, J. Am. Chem. Soc., 2010, 132,15459-15461.

8. M. Lelli, D. Gajan, A. Lesage, M. A. Caporini, V. Vitzthum, P. Miéville, F. Héroguel, F. Rascón, A. Roussey, C. Thieuleux, M. Boualleg, L. Veyre, G. Bodenhausen, C. Copéret and L. Emsley, J. Am. Chem. Soc., 2011, 133, $2104-2107$.
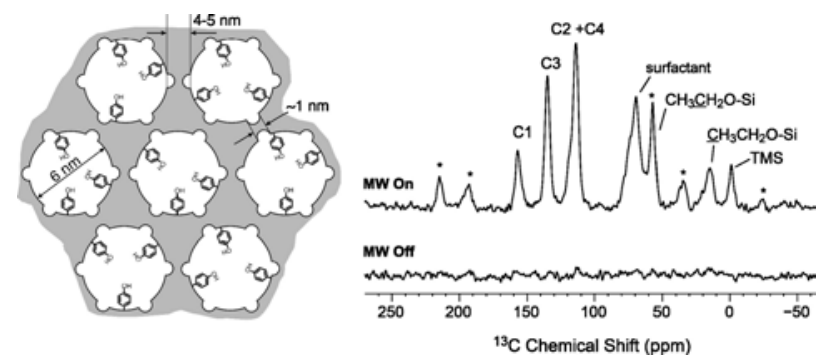

Figure 2. (from Ref. 8) Further characterization of functional groups on silica surfaces can be seen through ${ }^{1} \mathrm{H}-{ }^{29} \mathrm{Si}$ correlations. With routine DNP enhancements, fast 2D spectra can be acquired to determine the interactions of the functional group with the surface or lack thereof.
Figure 1. (adapted from Ref. 7) The structure of nanoporous silica functionalized with phenol groups is depicted in the schematic (left). The DNP solvent solution containing TOTAPOL is absorbed by the pores, resulting in DNP enhancements of 30 or more as shown by the ${ }^{13} \mathrm{C}$ crosspolarization spectra (inset).
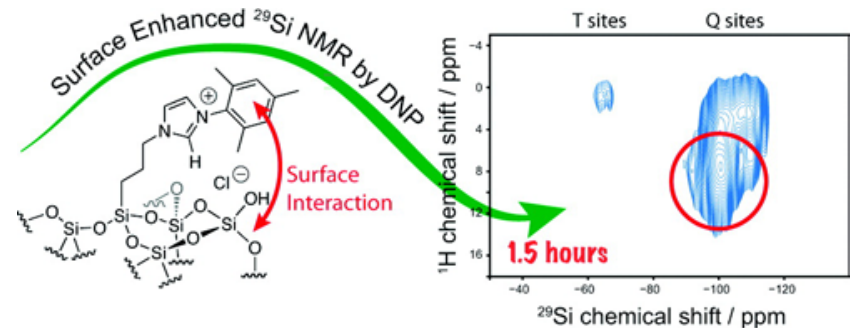


\title{
Solid-State DNP Spectrometer for Applications to Biological Solids and Material Science
}

\author{
Melanie Rosay ${ }^{1}$, Shane Pawsey ${ }^{1}$, Leo Tometich ${ }^{1}$, Richard J. Temkin ${ }^{2}$, Robert G. Griffin ${ }^{2}$, Werner E. Maas ${ }^{1}$ \\ ${ }^{1}$ Bruker BioSpin Corporation, Billerica, MA 01821; ${ }^{2}$ Massachusetts Institute of Technology, Cambridge, MA 02139 \\ E-mail: Melanie.Rosay@bruker-biospin.com
}

Dynamic Nuclear Polarization (DNP) experiments transfer the high Boltzmann polarization of unpaired electron spins to nuclear spins for large gain in sensitivity and dramatic reduction in signal averaging time. This sensitivity gain opens the door to applications in solid-state NMR that may not have been achievable otherwise ${ }^{1,2}$. The polarization transfer is driven by microwave irradiation of the electron spins near their Larmor frequency. For DNP experiments at ${ }^{1} \mathrm{H}$ frequencies in the range of $400-800 \mathrm{MHz}$, microwave sources operating in the range of 263-527 GHz are required with high output power, frequency, power stability, and reliability.

We report on the development of a DNP spectrometer for solid-state NMR applications with a microwave frequency of $263 \mathrm{GHz}, 400 \mathrm{MHz}{ }^{1} \mathrm{H}$ frequency. Microwaves are generated with a continuous-wave gyrotron and transmitted to the NMR sample via corrugated waveguide. The sample is then irradiated for DNPNMR experiments while spinning at the magic angle in a 3.2-mm rotor ${ }^{3}$. DNP signal enhancements of up to a factor of 80 at $100 \mathrm{~K}$ using the biradical TOTAPOL ${ }^{4}$ have been measured, and a broad range of samples have been successfully polarized, spanning from small molecules to large biological complexes and materials. We will describe the spectrometer design and show

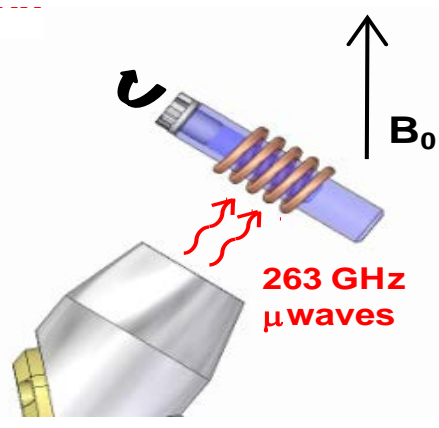
applications examples. Future directions will also be discussed, including higher frequency DNP spectrometers for sensitivity-enhanced NMR experiments at 600 and $800 \mathrm{MHz}{ }^{1} \mathrm{H}$ frequency.

\section{References}

1. $\quad$ L. R. Becerra et al., Phys. Rev. Lett., 22, 3561 (1993);

M.L. Mak-Jurkauskas et al., Proc. Natl. Acad. Sci., 105, 883 (2008).

2. A. Lesage et al., J. Am. Chem. Soc., 132, 15459 (2010).

3. C. Song et al., J. Am. Chem. Soc., 128, 11385 (2006).

4. M. Rosay, et al., Phys. Chem. Chem. Phys., 12, 5850 (2010). 


\title{
Next-Generation DNP-NMR Instrumentation
}

\author{
Thorsten Maly and Jagadishwar Sirigiri \\ Bridge12 Technologies, Inc., 37 Loring Drive, Framingham, MA 01702-8768 \\ E-mail: tmaly@bridge12.com; jsirigiri@bridge12.com
}

DNP-enhanced NMR spectroscopy has been demonstrated to yield signal enhancement factors up to several hundred at high magnetic fields for a range of nuclei. The technique has the potential to revolutionize the role of NMR spectroscopy in many research areas, such as structure determination of bio-macromolecules and material science. DNP using highpower microwave sources at high magnetic fields has been investigated in laboratory setups for almost two decades, but commercial instruments have only been recently available to the general NMR community. In this presentation, we will discuss the instrumentation necessary for DNP spectroscopy and the latest advances in gyrotron technology for DNPNMR applications being pursued at Bridge12. We also will present details on a novel, user-friendly, modular and economical DNP-NMR system currently under development at Bridge12. 



\section{Workshop Participants}

\section{Co-Organizers}

Karl Mueller

Marek Pruski

\section{External Participants}

Marc Caporini

Ulla Gro Nielsen

Song-I Han

John Hanna

Christian Hilty

Cynthia Jenks

Takeshi Kobayashi

Olivier Lafon

Thorsten Maly

Melanie Rosay

David Singel

Jagadishwar Sirigiri

Mark Smith

Kent Thurber
Pacific Northwest National Laboratory

Ames Laboratory

\section{Bruker Biospin}

University of Southern Denmark

University of California, Santa Barbara

University of Warwick

Texas A\&M University

Ames Laboratory

Ames Laboratory

Ecole Nationale Superieure de Chimie de Lille

Bridge12 Technologies Inc.

Bruker Biospin

Montana State University

Bridge12 Technologies Inc.

University of Warwick

National Institutes of Health

\section{EMSL/PNNL Participants}

Josh Adkins

Donald Baer

Suresh Baskaran

Garry Buchko

Sarah Burton

Allison Campbell

Herman Cho

John Cort

Greg Exarhos

Andy Felmy

Bruce Garrett

Carol Hirschmugl

David Hoyt

Jianzhi Hu

Nancy Isern

Bernd Kabius
David Koppenaal

Jinfeng Lai

Johannes Lercher

Andrew Lipton

Paul Majors

Hardeep Mehta

Chuck Peden

Jesse Sears

Wendy Shaw

William Shelton

Tom Squier

Raymond Teller

Eric Walter

Hongfei Wang

Yong Wang

Nancy Washton

Steven Wiley 



\section{Appendix C}





\section{Workshop Agenda}

\section{Science Drivers and Technical Challenges for Advanced Magnetic Resonance}

\section{Tuesday, December 6, - Thursday, December 8, 2011}

Tuesday, December $6^{\text {th }}$

$6: 30-9: 30 p m$

Dinner by invitation only

Anthony's

Please meet at the Guest House

parking lot at 6:10pm

Wednesday, December $7^{\text {th }}$

8:00 - 8:45 AM

Welcome: Dr. Allison Campbell

EMSL 1077

8:45 - 9:00 AM

Introductions and Scope:

EMSL 1077

Dr. Karl T. Mueller (EMSL/PNNL)

Dr. Marek Pruski (Ames)

9:00 - 9:45 AM

Introductory Lecture on DNP:

EMSL 1077

Dr. Olivier Lafon (ENSCL)

Dynamic Nuclear Polarization at

High Magnetic Field: How Can

Electrons Align Nuclei?

$9: 45-10: 30$ AM

DNP Science Lecture 1:

EMSL 1077

Dr. Christian Hilty (TAMU)

Chemical and Biochemical

Reactions Investigated by

Dynamic Nuclear Polarization

$10: 30-11: 15$ AM

DNP Science Lecture 2:

EMSL 1077

Dr. Song-I Han (UCSB)

Opportunities of DNP for the study

of Interactions and Interfaces

in Solutions and Solids 
11:15 - 12:00 PM

12:00 - 12:30 PM

$12: 30-1: 30$ PM

$1: 30-2: 00 \mathrm{PM}$

2:00 - 2:45 PM

$2: 45-3: 30$ PM

$3: 30-5: 30$ PM

$5: 30-6: 30$ PM
DNP Science Lecture 3:

EMSL 1077

Dr. Mark Smith (Warwick):

Recent DNP Work at Warwick Investigating Factors Determining DNP Signal Enhancement from Liquids at $94 \mathrm{GHz}$ and MAS DNP NMR using a Quasioptical Microwave Transmission Design

Working Lunch for visiting team \& invited staff

EMSL 1077

EMSL 1077

Plenary Lecture (Brown Bag)

Dr. David Singel (Montana):

Starburst Dendrimers with

Tailored Spin Labeling for DNP at High Fields

DNP Science Lecture 4:

EMSL 1077

Dr. Olivier Lafon (ENSCL)

High-field ${ }^{29} \mathrm{Si},{ }^{13} \mathrm{C}$ and ${ }^{27} \mathrm{Al}$

Dynamic Nuclear Polarization of

Porous Materials and

Nanoparticles

DNP Science Lecture 5:

EMSL 1077

Dr. Kent Thurber (NIH)

DNP with MAS at 9 Tesla and 25

$K$ : Theoretical and Experimental

DNP Science Lecture 6:

Dr. Marc Caporini (EPFL)

Extending Applications of Solid-

state MAS-DNP NMR to New

Systems and Nuclei

EMSL 1077

Breakout Sessions 1:

Material Sciences (talk by Greg

EMSL 1077

Exarhos)

Biological Sciences (talk by Steve Wiley)

EMSL Boardroom

Tour of EMSL Magnetic

EMSL LABS

Resonance Capability

Dinner (by invitation)

Dr. Bills Bistro 
Thursday, December 8, 2011

$8: 00-10: 30$ am

10:30 - 11:15 AM

11:15 - 12:00 PM

12:00 - 1:00 PM

1:00 - 3:00 PM

3:00 - 5:00 PM

5:00 PM
Breakout Sessions 2:

Catalysis Sciences (talk by Chuck

EMSL 1077

Peden)

Biogeochemical Sciences (talk by

Andy Felmy)

EMSL 1044

DNP Instrumentation Lecture 1:

EMSL 1077

Dr. Melanie Rosay (Bruker)

Solid-State DNP Spectrometer for

Applications to Biological Solids

and Material Science

DNP Instrumentation Lecture 2:

EMSL 1077

Drs. Jagadishwar Sirigiri and

Thorsten Maly (Bridge12)

Next Generation DNP-NMR

Instrumentation

Working Lunch (visitors and

EMSL 1077

invited guests)

Report Out from Breakout

EMSL 1077

Sessions

Catalysis Sciences

Biological Sciences

Materials Sciences

Biogeochemical Sciences

Writing Draft of Workshop Report

EMSL 1077 